\title{
Cuide-se para Cuidar: a promoção do autocuidado entre mães de crianças com deficiência por meio da extensão universitária
}

\author{
Self-care to Care: promoting self-care amongst mothers of disabled children through \\ university extension
}

\section{Cuida de Cuidar: la promoción del autocuidado entre las madres de niños con discapacidad a través de la extensión universitaria}

Gabriela Muniz Vidigal dos Santos ${ }^{1 *}$, Isabela Araújo Pícoli ${ }^{1}$, Ana Luiza Salgado ${ }^{1}$, Ana Carolina Domingues Ferreira ${ }^{1}$, Carla de Paula Silveira ${ }^{1}$, Isabela Mie Takeshita ${ }^{1}$.

\section{RESUMO}

Objetivo: Relatar a experiência de acadêmicas de Fisioterapia, Medicina, Enfermagem e Psicologia na realização do projeto de extensão "Cuide-se para Cuidar", cujo objetivo era promover o autocuidado entre mães de crianças com deficiência. Relato de experiência: Durante 6 semanas, o projeto de extensão "Cuidese para Cuidar" foi realizado e teve como metodologia rodas de conversa virtuais sobre autocuidado com mães de crianças com deficiência. A fim de promover qualidade de vida dessas mães, foram abordados aspectos sobre saúde mental e física, e assuntos diversos focados no bem-estar dessas mulheres, tais como autoestima, relacionamento conjugal, qualidade de sono e organização da rotina. Considerações finais: Foi possível observar a complexidade biopsicossocial das mães de crianças com deficiência. A extensão universitária possibilitou a compreensão da realidade da rotina, muitas vezes exaustiva, das cuidadoras que dedicam integralmente para o cuidado dessas crianças. O projeto proporcionou, portanto, o protagonismo das mães em seu autocuidado e valorizou sua experiência enquanto sujeitos concretos para a promoção da saúde.

Palavras-chave: Mães, Crianças com deficiência, Relações comunidade-instituição, Autocuidado, Promoção da saúde.

\section{ABSTRACT}

Objective: To report the experience of students of Physiotherapy, Medicine, Nursing and Psychology in the execution of the extension project "Cuide-se para Cuidar", with the goal to promote self-care among mothers of disabled children. Experience report: For a period of 6 weeks, the extension project "Cuide-se para Cuidar" was executed through the methodology of virtual conversation circles about self-care with mothers of disabled children. In order to promote these mother's quality of life, important topics about mental and physical health, and other themes related to well-being were highlighted, such as self-esteem, marital relationship, quality of sleep and organization. Final considerations: It was possible to observe the biopsychosocial complexity of mothers who have children with disabilities. University extension made it possible to understand the reality of the routine, often exhausting, of caregivers who fully dedicate themselves to the care of these children. The project provided, therefore, the role of mothers in their self-care and valued their experience as concrete subjects of their own health promotion.

Keywords: Mothers, Disabled children, Community-institutional relations, Self care, Health promotion.

\section{RESUMEN}

Objetivo: Informar la experiencia de los estudiantes de Fisioterapia, Medicina, Enfermería y Psicología en la realización del proyecto de extensión "Cuide-se para Cuidar", cuyo objetivo fue promover el autocuidado entre las madres de niños con discapacidad. Informe de experiencia: Así, por un período de 6 semanas, el proyecto de extensión "Cuide-se para Cuidar" tuvo como metodología la realización de círculos virtuales de conversación sobre autocuidado con madres de niños con discapacidad. Con el fin de promover la calidad de vida de estas madres, se abordaron aspectos de salud mental y física y diversos temas enfocados al bienestar

${ }^{1}$ Faculdade Ciências Médicas de Minas Gerais, Belo Horizonte - MG. *E-mail: gabriela.munizv@gmail.com 
de estas mujeres, como la autoestima, la relación conyugal, la calidad del sueño y la organización de la rutina. Consideraciones finales: Se pudo observar la complejidad biopsicosocial de las madres de niños con discapacidad. Además, la extensión universitaria permitió comprender la realidad de la rutina, muchas veces agotadora, de los cuidadores que se dedican de lleno al cuidado de estos niños. El proyecto aportó, por tanto, el papel de las madres en su autocuidado y valoró su experiencia como sujetos concretos para la promoción de la salud.

Palabras clave: Madres, Niños con discapacidad, Relaciones comunidad-institución, Autocuidado, Promoción de la salud.

\section{INTRODUÇÃO}

A descoberta de um diagnóstico de deficiência em uma criança afeta profundamente a dinâmica de sua família (MALEKSHAHI F, et al., 2020). Crianças com deficiência demandam cuidados contínuos, o que pode gerar condições estressantes e sobrecarga para seus familiares cuidadores, o que compromete seu cotidiano, gerando prejuízos às atividades diárias, ao trabalho e à própria subjetividade desses indivíduos, com impactos na autoestima e nos relacionamentos (SANTOS MA e MARTINS MP, 2015; YARAR F, et al., 2020). Tais impactos afetam especialmente as mães, uma vez que elas são as principais cuidadoras de crianças com deficiência (BARBOSA TA, et al., 2015).

A sobrecarga das mães no cuidado a uma criança com deficiência potencializa sintomas depressivos, como estresse, insônia, dificuldade nas relações interpessoais e ansiedade (CHU J e RICHDALE A, 2009). Isso implica no fato de que elas apresentam maior probabilidade de ter problemas de saúde mental do que 0 restante da população (PARK E e KIM J, 2019).

Os diversos tipos de deficiência afetam de forma diferente a rotina de cada família, assim como crianças com Síndrome de Down possuem necessidades diferentes daquelas com autismo e, por isso, deve-se considerar as singularidades de cada sistema familiar ao propor medidas para a qualidade de vida (SILVA S e PONTES F, 2016; SPINAZOLA C, et al., 2018).

A qualidade de vida das mães pode influenciar na criação de seu filho com deficiência e tende a ser menor do que a de mães de crianças sem deficiência, o que reitera a necessidade de programas terapêuticos que atuem especificamente sobre esse cenário (FEREIDOUNI Z, et al., 2021).

A extensão universitária, pode ser uma ferramenta neste processo, ela visa interação entre comunidade acadêmica e sociedade, incentivando a atuação de acadêmicos no enfrentamento de questões da sociedade e produção de mudanças voltadas ao desenvolvimento social (MINISTÉRIO DA EDUCAÇÃO, 2018).

Este artigo teve como objetivo relatar a experiência de acadêmicas dos cursos de Enfermagem, Fisioterapia, Medicina e Psicologia na execução do projeto de extensão "Cuide-se para Cuidar" vinculado a uma Instituição de Ensino Superior (IES) privada de Minas Gerais, que visava a promoção do autocuidado entre mães de crianças com deficiência.

\section{RELATO DE EXPERIÊNCIA}

Este relato de experiência é resultado do projeto de extensão intitulado "Cuide-se para Cuidar" cuja proposta foi promover o autocuidado das mães de crianças com deficiência por meio de rodas de conversa virtuais. O processo seletivo foi disponibilizado no site da IES e foram selecionadas oito acadêmicas dos cursos de Enfermagem, Medicina, Fisioterapia e Psicologia, com base em respostas a cinco questões sobre extensão universitária e suas repercussões. O projeto ocorreu entre março e julho de 2021. Foram realizadas reuniões de planejamento e rodas de conversa pela plataforma Google Meet $₫$ e foram elaborados materiais de divulgação e apresentação na plataforma Canva ${ }^{\circledR}$.

As rodas de conversa foram escolhidas por serem orientadas pela Educação Popular que promove a educação em saúde baseada na troca de saberes, valoriza vivências, cognição, subjetividade e a concretude de seus participantes (SAMPAIO J, et al., 2014). Metodologia assertiva para valorizar o protagonismo das mães em seu autocuidado. 
Foi necessário elaborar uma logo em parceria com um designer (Figura 1) que correspondesse à mensagem de acolhimento que desejava-se passar para as mães. Foi elaborado um vídeo como forma de convite aos encontros, este continha frases de efeito como: "Autocuidado não é egoísmo", "Para cuidar de quem você ama, primeiro você precisa estar bem consigo mesma" e "Apaixone-se por cuidar de si mesma".

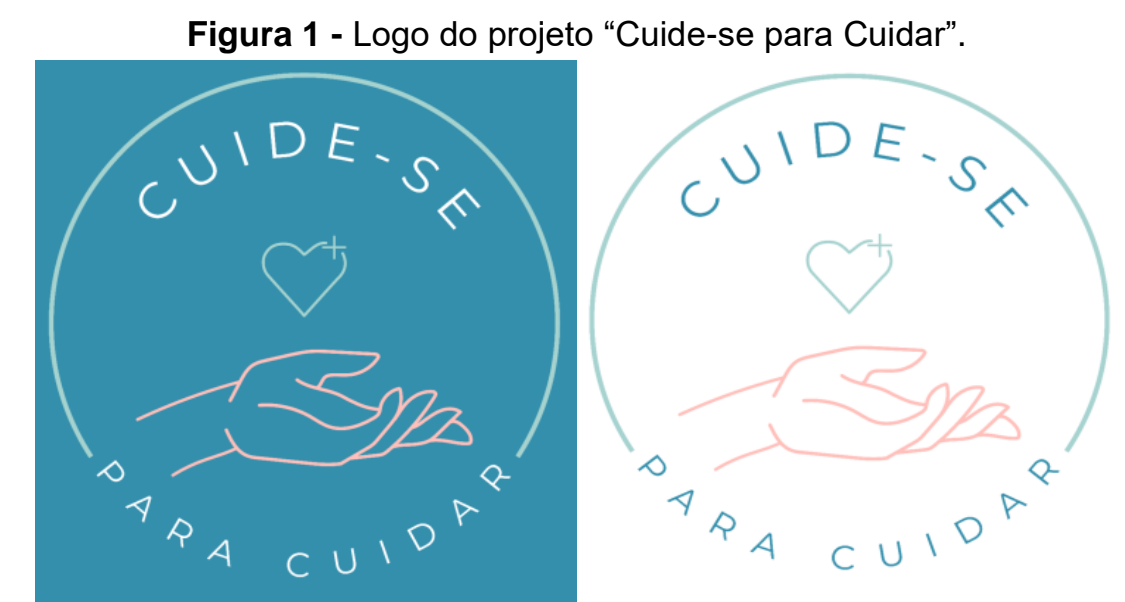

Nota: $A$ imagem foi criada e elaborada pelo designer através da plataforma Canva ${ }^{\circledR}$.

Fonte: Santos GMV, et al., 2021. A ilustração foi cedida por: Marcos Antonino França Volpi, 2021.

As mães eram contatos prévios das professoras orientadoras. Aquelas interessadas em participar das rodas de conversa preencheram um formulário por meio da plataforma Google Formulários $\circledast$ contendo questões fechadas e abertas. O formulário continha perguntas pessoais, como raça, escolaridade, estado civil da mãe e perguntas específicas sobre a patologia da criança e se a mãe possui uma rede de apoio, além de questionamentos referentes à disponibilidade e a temas de interesse para serem abordados nas rodas de conversa.

Os dados do questionário foram analisados para que as participantes pudessem ser adicionadas a grupos de WhatsApp de acordo com os dias da semana e horários que elas estariam disponíveis para a participação das rodas de conversa. Os dias e horários disponibilizados quinzenalmente foram: terça-feira às 17 ou 18 horas e quarta-feira às 16 ou 17 horas. O tempo estimado para cada reunião foi de trinta minutos a uma hora. Os convites de cada encontro, os links para o acesso às reuniões e os materiais de apresentação elaborados no Canva ${ }^{\circledR}$ eram enviados pelo Whatsapp ${ }^{\circledR}$.

No total, dezenove mães participaram dos encontros. Cada horário contou com a participação de 3 a 6 mães inicialmente. Após dois encontros, os horários de terça-feira 17 horas e quarta-feira 16 horas tiveram baixa participação, com duas mães e nenhuma, respectivamente. As interessadas foram realocadas para os horários de terça-feira 18 horas e quarta-feira 16 horas e até a conclusão do projeto esses grupos contaram com a participação de oito mães ao todo.

Para cada encontro foram elaborados slides contendo imagens, perguntas e informações. Estes ajudaram a incitar a reflexão das mães sobre os assuntos abordados, convidando-as a compartilhar suas experiências, promovendo discussão. Foram inseridas referências culturais como podcasts e vídeos, cartilhas informativas e informações em saúde baseadas em evidências. O papel das acadêmicas durante as rodas de conversa foi sobretudo de mediação, convidando as mães a compartilharem suas reflexões sobre cada tema e apresentando o material visual elaborado para o encontro.

As oito alunas participantes do projeto de extensão foram subdivididas em dois grupos, cada um contendo quatro integrantes. Neste relato serão discutidas apenas as rodas de conversa promovidas pelas autoras, aquelas participantes de um dos grupos. Os temas abordados nesses encontros foram: Formas de organizar a rotina diária e ter tempo para si mesma; técnicas para relaxamento e sono adequado; autoestima e relacionamento conjugal. 


\section{DISCUSSÃO}

O primeiro encontro abordou a rotina. A apresentação iniciou com uma dinâmica de perguntas reflexivas sobre a percepção do tempo e como ele era utilizado. As mães relataram suas experiências sobre a rotina e seu desejo de disponibilidade de tempo para realizar atividades de autocuidado. As respostas variaram, mas a predominante foi que seria necessário um dia mais longo para que fosse possível fazer todas as tarefas necessárias.

A rotina tem um papel importante no cotidiano, assim como uma rede de apoio adequada para as mães especiais (SANTOS MA e MARTINS MP, 2015). No encontro foi abordada a importância de saber delegar funções, uma ação que pode proporcionar às mães mais tempo para realizar outros afazeres e diminuir sua sobrecarga. Existe uma desigualdade intrafamiliar que contribui para que seja imposta sobre as mulheres a obrigação de realizar as tarefas domésticas e cuidados com o filho, o que as atrapalha de ter liberdade de exercer outras atividades (DUARTE G e SPINELLI LM, 2019). Foram apresentadas dicas de instrumentos para organização, como o Google Agenda® e "planners". Muitas participantes relataram que não planejavam suas tarefas diárias, enquanto outras já tinham métodos pessoais de organização e compartilharam sua experiência positiva.

Ao longo dos encontros subsequentes, as acadêmicas questionaram se as dicas e reflexões estavam sendo colocadas em prática. As mães que passaram a adotar os métodos de planejamento relataram maior produtividade e sentiram-se mais realizadas. Assim, os benefícios de planejar a rotina ficaram claros, uma vez que o planejamento possibilita uma diminuição da sobrecarga mental e da ansiedade (BITTENCOURT JA, et al., 2013).

Esse encontro foi de suma importância para que as acadêmicas pudessem iniciar a construção do vínculo com as mães participantes. A preparação para o encontro englobou um estudo sobre o tema e também uma elaboração conjunta da apresentação. Assim, houve aprendizado sobre como a colaboração entre os diferentes cursos da saúde pode enriquecer ações educativas.

O segundo encontro teve como temática técnicas de relaxamento e sono de qualidade. As mães foram questionadas sobre o que seria necessário para ter uma boa noite de descanso. A identificação e funcionamento das fases do sono foram abordadas e questionou-se as mães sobre sua quantidade média de horas de sono por noite. As respostas foram parecidas, relatando dormir menos de oito horas por noite, ou seja, menos do que a quantidade recomendada para a faixa etária adulta (HIRSHKOWITZ P, et al., 2015). A higiene do sono foi abordada e exemplificada a importância de uma rotina noturna para toda a família, revelando a correlação entre os temas rotina e sono.

Uma das alunas conduziu uma meditação guiada durante cinco minutos para relaxamento do grupo. As alunas perguntaram às mães quais atividades elas realizavam com frequência antes de dormir, para identificar hábitos como o uso excessivo de celular e televisão, os quais afetam diretamente a qualidade do sono (FREITAS CCM, 2017). Os relatos demonstraram que a maioria usa esses dispositivos antes de dormir. Uma das mães comentou que dorme com a televisão ligada e no outro dia não acorda bem disposta.

Foram passadas dicas para a melhoria do sono e técnicas de relaxamento, dentre elas, a aromaterapia, eficiente para melhorar a qualidade do sono. A inalação dos aromas pode promover a sensação de tranquilidade (DOMINGOS TS e BRAGA EM, 2014). Esse método foi corroborado pelas próprias mães, algumas já utilizavam dessa prática e compartilharam sua experiência com as outras participantes. Foi ressaltada pelas acadêmicas a importância de consultar um profissional da área para utilização adequada.

Houve maior espontaneidade e criatividade, por ser o segundo encontro, com uso de dinâmicas, como a meditação guiada. Além disso, foi possível dar exemplos bem direcionados às demandas das mães, ao recomendar a redução do uso do telefone antes de dormir.

O terceiro encontro teve como tema a autoestima e o relacionamento conjugal. No início desse encontro as mães foram convidadas a refletir sobre o que mais gostam sobre si e se a autoestima influencia suas atividades diárias. O nível de autoestima da mãe desempenha um papel essencial em sua adaptação ao processo de criação do filho, dessa forma, fazer as participantes da roda de conversa refletir sobre sua autoestima é um modo de fazê-las pensar sobre suas vivências na maternidade (SANTOS MA e MARTINS 
MP, 2016). As mães relataram que com o avanço da idade passaram a gostar mais de atributos da personalidade e comportamento e menos de atributos físicos. Sobre o impacto da autoestima nas atividades diárias, elas relataram que se sentir bem consigo mesma impacta diretamente em seu ânimo para exercer as tarefas e no modo com que se relacionam com a família.

As mães foram questionadas sobre seus relacionamentos familiares e romântico-afetivos. Muitas relataram que o nascimento do filho afetou o relacionamento conjugal, devido ao cansaço e a falta de sono nesse período. Com a maternidade, a percepção de si enquanto mulher e pessoa mudou, houveram vários relatos de crises de identidade durante os primeiros meses após o nascimento dos filhos. As participantes reiteraram que não são mães sozinhas e refletiram que a atuação do pai não é apenas uma ajuda, mas sim uma obrigação, elas sentem que é inevitável que o cuidado maior recaia sobre a mãe. Ao fim dessa discussão foram apontados hábitos para um relacionamento saudável e as mães compartilharam suas experiências positivas como: manter o diálogo para resolução de conflitos e situações e ter um momento sozinha com o companheiro.

A saúde sexual foi abordada ressaltando a importância do respeito e da intimidade nas relações e trazendo temas como masturbação e prazer feminino. O sexo e a sexualidade são tratados como tabus e frequentemente abordados de forma higienista, abordando prevenção de doenças e contracepção (FILHO VGS, 2019). Somente uma das mães quis participar nesse momento e contou sobre como a cumplicidade com seu parceiro auxilia na construção de uma vida sexual prazerosa para ambos. Observou-se desconforto das demais participantes quando convidadas a participar. Também houve um momento de fala sobre violência doméstica em que foram discutidas a legislação vigente sobre o tema e os canais de denúncia existentes.

Durante a elaboração desse encontro as acadêmicas sentiram-se mais apreensivas pois consideraram desafiador abordar o tema de modo respeitoso e efetivo. Por exemplo, ao falar de sexualidade e violência doméstica foi preciso superar o próprio desconforto e preparar-se para receber reações negativas ou indiferentes das mães.

Após cada encontro as acadêmicas se reuniam com as orientadoras para uma auto avaliação e para planejar a próxima roda de conversa. Por meio dessas reuniões constatou-se a importância de trazer dicas que as mães pudessem utilizar, e, principalmente, incentivá-las a compartilhar suas experiências. Os benefícios das reuniões em grupo para as mães de crianças com deficiência ocorrem através do compartilhamento de histórias e vivências com as quais as integrantes se identificam e começam a praticar as dicas relatadas (FONSECA S, et al., 2020). Esses relatos também contribuem na visão das acadêmicas dessa realidade, com melhor planejamento das atividades de extensão e aprimoramento na sua formação profissional e humanística.

Durante os encontros foi possível compreender como é o itinerário das mães pelo sistema de saúde e como os profissionais de saúde podem contribuir positiva ou negativamente. Dessa maneira, as alunas puderam aprender de modo único como é a realidade das famílias de crianças com deficiência e obtiveram espaço para desenvolver habilidades como: colaboração entre uma equipe multidisciplinar de trabalho, a pesquisa de evidências de qualidade sobre os temas apresentados às mães e a mediação de rodas de conversa. Assim, é evidente a necessidade de existirem projetos de extensão para contribuir com a formação acadêmica, profissional e pessoal dos indivíduos envolvidos, o que traz repercussões tanto os acadêmicos e professores das IES quanto a população-alvo (FILHO VGS, 2019).

A dificuldade em engajar as mães foi atribuída à dificuldade das acadêmicas em desenvolver estratégias efetivas de divulgação dos encontros e à dificuldade das mães em encontrar tempo para participar, portanto esses aspectos necessitam de aprimoramento na realização de atividades futuras.

Observa-se, portanto, que a realização do projeto "Cuide-se para Cuidar" cumpriu com os objetivos propostos. A execução do projeto possibilitou o desenvolvimento de habilidades essenciais à prática profissional futura das estudantes e a escuta qualificada das necessidades específicas das mães, fizeram com que elas se sentissem mais acolhidas e pudessem expressar seus sentimentos perante os tópicos abordados. Espera-se que a partir deste relato novas práticas extensionistas direcionadas a esse público-alvo sejam desenvolvidas e valorizadas. 


\section{AGRADECIMENTOS E FINANCIAMENTO}

Agradecemos a Faculdade Ciências Médicas de Minas Gerais por viabilizar este projeto de extensão universitária. Agradecemos as participantes, por disponibilizarem uma parte do seu tempo para entrarem nas reuniões e compartilharem suas dificuldades e alegrias durante o processo. Por fim, ao designer Marcos Antonino França Volpi por ter proporcionado ao projeto uma identidade visual.

\section{REFERÊNCIAS}

1. BARROS MB, et al. Quality of sleep, health and well-being in a population-based study. Revista de Saúde Pública, 2019; 53: 82.

2. BARBOSA TA, et al. Rede de apoio e apoio social às crianças com necessidades especiais de saúde. Rev Rene, 2016; 17(1): 60-6.

3. CHU J, RICHDALE AL. Sleep quality and psychological wellbeing in mothers of children with developmental disabilities. Research in developmental disabilities, 2009; 30(6): 1512-1522.

4. DOMINGOS TS, BRAGA EM. Significado da massagem com aromaterapia em saúde mental. Acta paulista de Enfermagem. 2014; 27(6): 579-84.

5. DUARTE G, SPINELLI LM. Estereótipos de Gênero, Divisão Sexual do Trabalho e Dupla Jornada. Iniciação Científica, 2019; 32(2): 126-45.

6. FEREIDOUNI Z, et al. A comparative study on the quality of life and resilience of mothers with disabled and neurotypically developing children in Iran. Heliyon, 2021; 7(6): e07285.

7. FILHO VGS. Paradigmas norteadores da história da educação sexual no Brasil: nas pegadas do higienismo, do conservadorismo religioso e da defesa dos direitos humanos. Revista Eletrônica de Teologia e Ciências das Religiões, 2019; 7(2): 159-76.

8. FONSECA SC, et al. Investigação-ação com mães de pessoas com deficiência intelectual: a redução da sobrecarga como um projeto de vida. Rev Educação Especial, 2020; 33:1-21.

9. FREITAS CCM, et al. Relação entre uso do telefone celular antes de dormir, qualidade do sono e sonolência diurna. Revista de Medicina, 2017; 96(1):14-20.

10. GUERRA CS, et al. Do sonho à realidade: experiência de mães de crianças com deficiência. Texto contexto Enfermagem., 2015; 24(2): 459-466.

11. HIRSHKOWITZ P, et al. National Sleep Foundation's sleep time duration recommendations: methodology and results summary. Journal of the National Sleep Foundation's, 2015; 1(1):40-43.

12. JURADO- FASOLI L, et al. Exercise training improves sleep quality: A randomized controlled trial. European journal of clinical investigation, 2020; 50(3): e13202.

13. MALEKSHAHI F, et al. Intellectually Disabled Children and Their Parents' Problems: Preliminary Evaluation and the Suggestion of Effective Strategies. Crescent Journal of Medical and Biological Sciences, 2020; 7(3): 336-341.

14. MINISTÉRIO DA EDUCAÇÃO. Conselho Nacional de Educação, Câmara de Educação Superior. Resolução № 7, de 18 de dezembro de 2018. Estabelece as Diretrizes para a Extensão na Educação Superior Brasileira e regimenta 0 disposto na Meta 12.7 da Lei no 13.005/2014, que aprova o Plano Nacional de Educação - PNE 2014-2024 e dá outras providências. $\quad$ Brasil, 2018. https://normativasconselhos.mec.gov.br/normativa/view/CNE_RES_CNECESN72018.pdf?query=revogacao. Acesso em: 02 de setembro de 2021.

15. NOGUEIRA IC, et al. Efeitos do exercício físico no controle da hipertensão arterial em idosos: uma revisão sistemática. Revista Brasileira de Geriatria e Gerontologia. 2012; 15(3): 587-601

16. SAMPAIO J, et al. Limites e potencialidades das rodas de conversa no cuidado em saúde: uma experiência com jovens no sertão pernambucano. Interface-Comunicação, Saúde, Educação, 2014; 18: 1299-1311.

17. SANTOS MA, MARTINS MP. Estratégias de enfrentamento adotadas por pais de crianças com deficiência intelectual. Ciência \& Saúde Coletiva, 2016; 21(10): 3233-44.

18. SILVA SSC, PONTES FAR. Rotina de famílias de crianças com paralisia cerebral. Educar em Revista, 2016; (59): 6578.

19. SPINAZOLA CC, et al. Children with Physical Disability, Down Syndrome and Autism: Comparison of Family Characteristics in the Maternal Perspective in Brazilian Reality. Revista Brasileira de Educação Especial, 2018; 24(2): 193-208.

20. PARK E, KIM J. Activity limitation in children with cerebral palsy and parenting stress, depression, and self-esteem: A structural equation model. Official journal of the Japan Pediatric Society, 2020; 62(4): 459-66.

21. PAVÃO MR, et al. Rotina e necessidades de apoio: relato de familiares de crianças de zero a dois anos, público alvo da Educação Especial. Revista Educação Especial, 2018; 31(61): 447-462.

22. UEDA R, et al. The quality of life of children with neurodevelopmental disorders and their parents during the Coronavirus disease 19 emergency in Japan. Scientific Reports, 2121; 11(1): 3042.

23. YARAR F, et al. Impact of having a disabled child on mothers' anxiety, depression and quality of life levels. Pamukkale TIp Dergisi, 2020; 14(1): 223-32. 